\title{
Visual Analysis of Nurse Rostering Solutions through a Bio-inspired Intelligent Model
}

\author{
$\mathrm{M}^{\mathrm{a}}$ Belen Vaquerizo, Álvaro Herrero \\ University of Burgos \\ Burgos, Spain \\ belvagar@ubu.es, ahcosio@ubu.es
}

\author{
Emilio Corchado \\ University of Salamanca \\ Salamanca, Spain \\ escorchado@usal.es
}

\begin{abstract}
In recent years, many solutions have been developed for the Nurse Rostering Problem. When a new problem of this kind arrives, it is not so easy to choose the proper solution from previous work. This study presents an application of a bioinspired intelligent system to analyse and select previous nurse rostering solutions. This applied research presents a multidisciplinary study based on the application of unsupervised neural projection models in order to identify the similar solutions to the mentioned problem. The system has been tested under a real data set gathered from the current state of the art, achieving promising results.
\end{abstract}

Nurse Rostering, Evolutionay Computation, Neural Projection Models, Unsupervised Learning

\section{INTRODUCTION}

Computational methods for rostering and staff scheduling have been a subject of continued research and commercial interest since the 1950s [7].

Nurse rostering is a complex scheduling problem that daily affects hospital personnel all over the world, and these types of problems have been an interesting subject for both artificial intelligence and operational research communities [4].

Real-life rostering problems are difficult combinatorial problems that belong to the domain of scheduling and timetabling, so these problems are considered as combinatorial-complexity scheduling problems. More specifically, they are classified as personnel scheduling problems, where a series of shifts and off-duties has to be allocated to each member of the workforce weekly. On the other hand, obtaining an optimal solution is an impracticable task to be carried out due to the large number of alternative schedules.

Plenty of different evolutionary solutions have been proposed to tackle these problems up to now. Thus, when trying to solve a new problem of this kind, it is not an easy task to select which of those previously applied solutions could be the most appropriate one. This study proposes unsupervised projection models to get a useful insight of previous evolutionary solutions to these problems, what leads to acquiring further knowledge about the state of the art. Additionally, the obtained projections can be also used to choose the solutions that best suits future similar problems.

\section{NURSE ROSTERING}

Personnel rostering problems [13, 15] are often highly constrained and difficult to be solved manually. Legal, management, and staff requirements are often conflicting and must be considered when taking rostering decisions.

\section{A. Problem Description}

There are some features of nurse rostering problems that are key to find the proper solution:

- Personnel: hospitals are organized in wards with fixed activities, usually a settled location, and, often, a permanent team of nurses.

- Skill categories: personnel members in a ward belong to one of several skill categories.

- Work regulations: cyclical schedules must obey very strict patterns.

- Shift types: a shift type is a predefined period with a fixed start and end time in which personnel members can be on or off duty. Many continuously working organisations schedule three typical shift types called morning, late and night shifts. Apart from locally redefining prototype shifts in a ward, exceptional shift characteristics are allowed for particular nurses.

- Planning period: planning periods for nurse rostering vary from a couple of days to a few months. The length of the period is expressed as a number of days or a number of assignment units.

- Schedule: the roster of the ward, in which the shift assignments to people are stored.

\section{B. Problem Constraints}

When allocating shifts, there might be several rules, preferences and requests to comply with. These constraints on the problem can be divided into two groups; those that are considered hard (must be taken into account in any solution) and those that are considered soft (can be relaxed if required to find a solution).

Usual constraints, both hard and soft, which are considered in this research are listed in Table 1. 
TABLE I. NURSE ROSTERING CONSTRAINTS

\begin{tabular}{|c|c|c|}
\hline$N^{o}$ & \multicolumn{2}{|c|}{ Categories of Hard Constraints } \\
\hline 1 & $A$ & Maximum one Assignment per Shift Type per Day \\
\hline \multirow[t]{2}{*}{2} & $B$ & Required Skill Category \\
\hline & $C$ & Personnel Requirements \\
\hline 4 & & Shift type requirements \\
\hline \multirow[t]{2}{*}{5} & & Floating requirements \\
\hline & $D$ & Hospital Constraints \\
\hline 6 & & Minimum time between two assignments \\
\hline \multirow[t]{2}{*}{7} & & Alternative skill category \\
\hline & $E$ & Constraints defined by the Work Regulation \\
\hline 8 & & Maximum number of assignments \\
\hline 9 & & Maximum number of consecutive days \\
\hline$N^{o}$ & \multicolumn{2}{|c|}{ Categories of Soft Constraints } \\
\hline 10 & & Minimum number of consecutive days \\
\hline 11 & & Maximum number of consecutive free days \\
\hline 12 & & Minimum number of consecutive free days \\
\hline 13 & & Maximum number of hours worked \\
\hline 14 & & Minimum number of hours worked \\
\hline 15 & & Maximum number of assignments per day of the week \\
\hline 16 & & Maximum number of assignments for each shift type \\
\hline 17 & & Maximum number of a shift type per week \\
\hline 18 & & Number of consecutive shift types \\
\hline 19 & & Assign two free days after night shifts \\
\hline 20 & & Assign complete weekends \\
\hline 21 & & No night shift before a free weekend \\
\hline 22 & & Assign identical shift types during the weekend \\
\hline 23 & & Maximum number of consecutive working weekends \\
\hline
\end{tabular}

It is often possible to consider a high number of soft constraints on the personnel schedules. Although they must be preferably satisfied, violations can be accepted to a certain extent. It is highly exceptional in practice to find a schedule that satisfies all the soft constraints.

The main target of the applied search algorithms is to minimise the real impact of violations of the constraints, previously specified by the users of the system.

Some of the constraints strengthen other constraints, while others are adverse factors in the planning of real-world hospital wards. Very often few constraints are even contradictory in real contexts. It is sometimes obvious that certain constraints can never be satisfied at all. In those cases, the user of the planning system must be informed about the extent to which each type of constraint is violated.
It is also necessary to consider the relaxations of some constraints due to holiday periods or sick leaves.

Boundary constraints (at the beginning and at the end of the planning period) have an important impact on the evaluation of the final solution. In general, the rule holds that a penalty is generated when a violation of a constraint could be avoided in the current planning period by appropriate scheduling. No violation is generated when the constraint, which is not satisfied, can still be satisfied by scheduling appropriate shifts in the next planning period.

\section{Description Of the StATE OF The ART}

The extensive collection of scientific literature and reviews of published papers by different reseachers [1, 2, 3, $4,11,12,14]$ on the topic of nurse rostering has been analysed in present study.

To describe the different solutions proposed so far to solve the rostering problem in an automated way, different features may be considered. A set of the most common features from the state of the art in this type of problems, are proposed in this paper and described in Table II.

\section{TABLE II. FEATURES OF NURSE ROSTERING SOLUTIONS}

\begin{tabular}{|c|c|}
\hline ID & Feature Description and possible values that can take \\
\hline $\mathrm{C} 1$ & $\begin{array}{l}\text { Applicability of the approach: not applied in practice but tested on } \\
\text { real data, applied in practice in just one hospital, or applied in } \\
\text { practice in several (more than one) hospitals. }\end{array}$ \\
\hline $\mathrm{C} 2$ & Coverage as considered as hard or soft constraint \\
\hline $\mathrm{C} 3$ & $\begin{array}{l}\text { Time related constraints for personnel as considered as hard or soft } \\
\text { constraints }\end{array}$ \\
\hline $\mathrm{C} 4$ & $\begin{array}{l}\text { Approach of optimization: minimise violations of time related } \\
\text { constraints, minimise violations of coverage and time related } \\
\text { constraints, minimise number of employees,minimise personnel } \\
\text { cost, minimise non-negative 'under' coverage, uniform distribution } \\
\text { of shortages and surpluses over weekdays, or minimise desviation } \\
\text { between scheduled people and the total work capacity from the } \\
\text { work regulations. }\end{array}$ \\
\hline $\mathrm{C} 5$ & $\begin{array}{l}\text { Approach of heuristic: minimise violations of time related } \\
\text { constraints, minimise violations of coverage and time related } \\
\text { constraints, minimse violations of coverage constraints, minimise } \\
\text { number of employees, minimise personnel cost, or minimise } \\
\text { desviation between scheduled nurses and demand. }\end{array}$ \\
\hline C6 & Flexibility on constraints: fixed, adaptable, or user-definable. \\
\hline C7 & Flexibility on costs and weights: fixed, adaptable, or user-definable) \\
\hline C8 & Approaches (Cyclical, Semy-cyclical, Non-cyclical) \\
\hline C9 & $\begin{array}{l}\text { Tackling coverage constraints: understaffing allowed or not } \\
\text { allowed. }\end{array}$ \\
\hline C10 & Tackling coverage constraints: overstaffing allowed or not allowed. \\
\hline C11 & $\begin{array}{l}\text { Tackling coverage constraints: other options different from } \\
\text { understanding and overstaffing are considered. }\end{array}$ \\
\hline C12 & $\begin{array}{l}\text { Number of skill categories that the models can handle: } 1,2,3 \text {, or } \\
\text { user-definable. }\end{array}$ \\
\hline $\mathrm{C} 13$ & $\begin{array}{l}\text { The way substitutability is organised: skill categories scheduled } \\
\text { separately, hierarchical substitutability, or user-definable } \\
\text { substitutability. }\end{array}$ \\
\hline C14 & $\begin{array}{l}\text { Flexibility in setting and defining work regulations: identical work } \\
\text { regulations for everybody, mixed workforce (full time and half time } \\
\text { nurses), user-definable work regulations, or float nurses (nurses } \\
\text { from different wards can solve personnel shortage). }\end{array}$ \\
\hline C15 & $\begin{array}{l}\text { Scope of usual changes in coverage constraints days, shifts, hours, } \\
\text { or a range between minimum and maximum coverage. }\end{array}$ \\
\hline C16 & $\begin{array}{l}\text { Flexibility regarding the definition of shift by type: strictly distinct } \\
\text { (off/on), overlapping allowed, strict start-end times, or floating } \\
\text { intervals. }\end{array}$ \\
\hline
\end{tabular}




\begin{tabular}{|c|c|}
\hline C & $\begin{array}{l}\text { ift or no shifts defined (days), three different shifts (usually } \\
\text { ferred to as morning, late, and night), defined length (the intervals } \\
\text { nnot be set by the users), or user-definable shifts (the number of } \\
\text { fferent shifts, their start and end times, and their length are set by } \\
\text { e users) }\end{array}$ \\
\hline C18 & $\begin{array}{l}\text { lenght: } 4 \text { days, } 1 \text { week, } 2 \text { weeks, } \\
\text { month, } 1 \text { year , or user defined. }\end{array}$ \\
\hline C19 & r-definable, fixed number or to be minimised. \\
\hline C20 & $\begin{array}{l}\text { Time related constraints: capacity (maximum number of } \\
\text { assignments), overtime (maximum number of assignments to a } \\
\text { particular shift type) or maximum number of shifts per week. }\end{array}$ \\
\hline C21 & $\begin{array}{l}\text { ts - finite capacity constraints: maximun } \\
\text {, overtime, maximun number of assignments } \\
\text { e., }\end{array}$ \\
\hline C22 & $\begin{array}{l}\text { preferen } \\
\mathrm{ft} \text { Patterns }\end{array}$ \\
\hline C23 & $\begin{array}{l}\text { Time related constraints - consecutiveness: maximum number of } \\
\text { consecutive days, minimum number of consecutive days, number of } \\
\text { consecutive days, maximum number of consecutive free days, } \\
\text { minimum number of consecutive free days, patterns (these are } \\
\text { cyclical constraints imposed on no-cyclical schedules), free days } \\
\text { after night shifts, no night shift before day off, time between } \\
\text { assignments, consecutive shifts, sequences of shift types, or mixture } \\
\text { of day-night shifts per week. }\end{array}$ \\
\hline C24 & eness: us \\
\hline C25 & the worklo \\
\hline C26 & d assignation: user-de \\
\hline C27 & $\begin{array}{l}\text { in } \mathrm{x} \\
\text { ation }\end{array}$ \\
\hline $\mathrm{C} 28$ & or set of values. \\
\hline C29 & $\begin{array}{l}\text { Time related constraints - other constraints not previously } \\
\text { described: preference days/nights, working history, changes in } \\
\text { shifts on consecutive days, maximum consecutive on/off/on } \\
\text { patterns, or people working together or not. }\end{array}$ \\
\hline
\end{tabular}

All these features have been analyzed and a value has been assigned to each one of them for every solution. As a result, a dataset has been generated as described in section V.

\section{BIO-INSPIRED INTELLIGENT MODELS}

Artificial Neural Networks are bio-inspired intelligent systems which emulate some of the features of real neural networks found in animal brains. The use of the word unsupervised declares that these networks will not be given a training regime which involves the use of pre-labelled data. This too models what happens in young animals: all animals must learn to identify structure in their environment in an unsupervised manner. It may be that supervised learning in animals will take place after the animal has reached a certain level of sophistication but certainly unsupervised learning must take place first. Learning in animals is thought to involve changes in the synapses with which neurons communicate. These synapses are modelled by parameters known as weights in the artificial intelligence community. Learning in artificial neural networks is through adaption, in some way, of the parameters of the network.

Patterns that exist across dimensional boundaries in high dimensional datasets may become visible if changes are made to the spatial coordinates. Projection models perform such change by projecting high-dimensional data onto a lower dimensional space in order to identify "interesting" directions in terms of any specific index or projection. Such indexes are the skew or kurtosis index in the case of Exploratory Projection Pursuit (EPP) [8]. Having identified the most interesting projections, the data is then projected onto a lower dimensional subspace plotted in 2D or 3D, which makes it possible to examine its structure with the naked eye.

To visually identify the structure and patterns in nurserostering dataset obtained from the literature review, two unsupervised neural models have been applied, namely Principal Component Analysis and Cooperative Maximum Likelihood Hebbian Learning. They are described in the following sub-sections.

\section{A. Principal Component Analysis}

Principal Component Analysis (PCA) [18, 19] is a standard statistical technique for compressing data; it can be shown to give the best linear compression of the data in terms of least mean square error. There are several Artificial Neural Networks (ANNs) or connectionist models which have been shown to perform PCA e.g. [9, 17].

This technique describes the variation in a set of multivariate data in terms of a set of uncorrelated variables, in decreasing order of importance, each of which is a linear combination of the original variables. Using PCA it is possible to find a smaller group of underlying variables that describe the data, with the result that the first few components of such a group might explain most of the variation in the original dataset.

It should be noted that even if we are able to characterize the data with a few variables, it does not follow that an interpretation will ensue.

\section{B. Cooperative Maximum Likelihood Hebbian Learning}

One neural implementation of EPP is MaximumLikelihood Hebbian Learning (MLHL) [5, 10], which identifies interestingness by maximising the probability of the residuals under specific probability density functions that are non-Gaussian. Cooperative Maximum Likelihood Hebbian Learning (CMLHL) [5, 6] is based on MLHL, adding lateral connections hich have been derived from the Rectified Gaussian Distribution [20]. The resultant net can find the independent factors of a data set but does so in a way that captures some type of global ordering in the data set.

Considering an $\mathrm{N}$-dimensional input vector $(x)$, and an M-dimensional output vector $(y)$, with $W_{i j}$ being the weight (linking input $j$ to output $i$ ), then CMLHL can be expressed as follows. Feed-forward step:

$$
y_{i}=\sum_{j=1}^{\mathbf{N}} W_{i j} x_{j}, \forall i
$$

Lateral activation passing:

$$
y_{i}(t+1)=\left[y_{i}(t)+\tau(b-A y)\right]^{+}
$$


Feedback step:

$$
e_{j}=x_{j}-\sum_{i=1}^{M} W_{i j} y_{i}, \forall j
$$

Weight change:

$$
\Delta W_{i j}=\eta \cdot y_{i} \cdot \operatorname{sign}\left(e_{j}\right)\left|e_{j}\right|^{p-1}
$$

Where: $\eta$ is the learning rate, $\tau$ is the "strength" of the lateral connections, $b$ the bias parameter, $p$ a parameter related to the energy function [6], [5] and $A$ is a symmetric matrix used to modify the response to the data [6].

The effect of the A matrix is based on the relation between the distances separating the output neurons.

\section{EXPERIMENTS \& RESULTS}

When a new rostering problem is to be solved, it would be helpful to automatically select an appropriate group of solutions applied to previous, similar problems. The experimental setup of this study is intended to show the effectiveness of some projection models in visualizing a dataset composed of previous nurse rostering solutions. Based on that, a previous solution applied to a similar problem can be easily selected.

\section{A. Dataset}

As stated in section III, several features from previous evolutionary solutions to the nurse rostering problem have been considered. Previous work has been analysed and each different proposed solution is included in the generated datasets as a new instance. Then, the corresponding values are assigned to each one of the 29 defining features (See Table II). The different values a feature may take have been quantified and assigned a number so that these features can be analysed by a neural network. Each one of these features takes the zero value when such feature is not present in the given solution.

As a result of the literature review, 1,686 different solutions by different authors are considered. Therefore, a dataset composed of 1,686 instances and 29 dimensions has been gathered.

\section{B. Results}

The previously described bio-inspired models have been applied to obtain a projection of the multidimensional dataset described in previous sub-section. Through the PCA and CMLHL projections (Figs. 1 and 2), different groups of solutions are obtained according to their similarity. Each one of the stars in the following figures (Figs 1 and 2) is associated to an instance (solution) from the analysed dataset.

The projection of the solution dataset obtained by means of PCA is shown in Figure 1. The shown two principal components account to $54,36 \%$ of the data variance, which is a low percentage of the whole dataset.

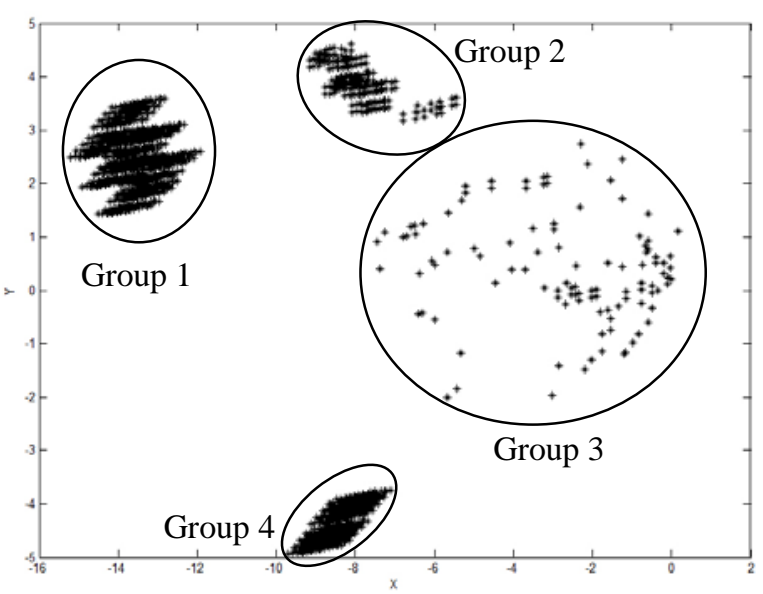

Figure 1. PCA Projection.

As it is shown in Fig. 1, four groups (from Group 1 to Group 4) of solutions can be differentiated and obtained through PCA. 864 of the analyzed instances are located in Group 1, 231 in Group 2, 159 in Group 3, and 432 in Group 4.

So, according to these four groups of solutions obtained through PCA, it can be said that some of the features (such as C3, C21, C22, C23 and C24) are present in the solutions from all the groups. This means that in most of the analyzed studies, the constraints related with the time can be considered as hard or soft, and there are others constraints such as finite capacity, personal preferences and the consecutiveness constraints that can be considered with certain values that could be defined by the user.

Depending on the features considered in the new rostering problem to solve, the next groups of solutions, shown in Table III (where the features are labeled in the first column as in Table II), can be studied or analyzed to solve that new problem.

TABLE III. GROUPS OF ROSTERING SOLUTIONS FROM PCA PROJECTION AND CONSIDERED FEATURES

\begin{tabular}{|l|c|c|c|c|}
\hline ID & Group 1 & Group 2 & Group 3 & Group 4 \\
\hline C1 & & & -- & \\
\hline C2 & $--(1)$ & $--(1)$ & -- & \\
\hline C3 & -- & -- & -- & -- \\
\hline C4 & $--(1)$ & $--(1)$ & -- & \\
\hline C5 & & & -- & \\
\hline C6 & $--(1)$ & $--(1)$ & -- & \\
\hline C7 & $--(1)$ & $--(1)$ & -- & \\
\hline C8 & & -- & -- & \\
\hline C9 & -- & -- & -- & \\
\hline C10 & $--(1)$ & $--(1)$ & & -- \\
\hline C11 & $--(1)$ & $--(1)$ & & -- \\
\hline C12 & & & -- & \\
\hline C13 & -- & -- & -- & \\
\hline C14 & -- & -- & -- & \\
\hline C15 & -- & -- & -- & \\
\hline C16 & $--(1)$ & $--(1)$ & -- & \\
\hline C17 & -- & -- & -- & \\
\hline C18 & -- & -- & -- & \\
\hline C19 & $--(1)$ & $--(1)$ & -- & \\
\hline C20 & $--(1)$ & $--(1)$ & -- & \\
\hline
\end{tabular}




\begin{tabular}{|l|c|c|c|c|}
\hline C21 & -- & -- & -- & -- \\
\hline C22 & -- & -- & -- & -- \\
\hline C23 & -- & -- & -- & -- \\
\hline C24 & -- & -- & -- & -- \\
\hline C25 & -- & -- & -- & \\
\hline C26 & $--(1)$ & $--(1)$ & -- & \\
\hline C27 & $--(1)$ & $--(1)$ & -- & \\
\hline C28 & $--(1)$ & $--(1)$ & & -- \\
\hline C29 & $--(1)$ & $--(1)$ & & -- \\
\hline
\end{tabular}

It must be said that the same features could be considered in different ways (taking different values) in the different groups as listed in Table I. That case is marked in that table as (1) for those groups in which they are considered in a different way.

The projection obtained through CMLHL is shown in Figure 2, and its explanation about the solutions grouped in the different groups obtained would be in the same way that with PCA Projection.

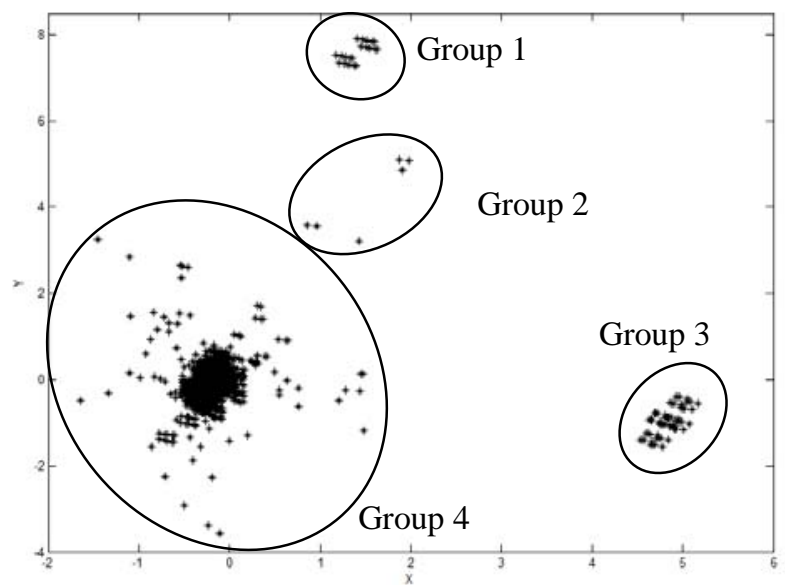

Figure 2. CMLHL Projection.

Moreover, as can be seen in Fig. 2, the different groups clustering the whole set of solutions are more clearly identified that in the case of PCA projection. That would help in selecting the best solutions to be applied in a new nurse rostering problem, given the features of the problem.

\section{CONCLUSIONS AND FUTURE WORK}

This paper presents a study about the different features considered in the extensive collection of scientific literature and reviews of published papers by different researchers on the topic of nurse rostering.

Through the PCA and CMLHL projections (Figs. 1 and 2), different groups of solutions are obtained according to the similarity of the analyzed features. As an outcome from these projections, previously applied solutions for the rostering problem can be visually grouped.

Each group in the projections includes rostering solutions by different authors, with certain common features considered in the treatment of this problem. The purpose of this idea is to facilitate the study of new rostering problems, where for each new problem to be solved, according to its features, the group or groups that collect these characteristics could be identified. Thus, it can be obtained a selection of previous solutions whose features are similar to the presented work.

Although the selection of previous nurse rostering solutions is not an easy task, it can be seen from present study that some bio-inspired models may be considered as useful tools to gain deep knowledge about those previous solutions. Consequently, those neural projection models can be applied to select the rostering solutions that best fit a new given problem.

Future work will apply some other projection/visualization models for a far more general comparative study. On the other hand, visualization models will be also combined with some other tools to obtain further knowledge about the analysed dataset.

\section{ACKNOWLEDGMENT}

This research is partially supported through the project of the Spanish Ministry of Education and Innovation CIT020000-2009-12 (funded by the European Regional Development Fund), project of the Spanish Ministry of Science and Innovation TIN2010-21272-C02-01 (funded by the European Regional Development Fund). The authors would also like to thank the vehicle interior manufacturer, Grupo Antolin Ingenieria S.A., within the framework of the project MAGNO2008 - 1028.- CENIT Project funded by the Spanish Government.

\section{REFERENCES}

[1] G, Beddoe and S, Petrovic, "Enhancing case-based reasoning for personnel rostering with selected tabu search". Journal of the concepts, vol. 58, Dec. 2007, pp. 1586-1598.| doi:10.1057/palgrave.jors.2602304.

[2] G. Beddoe, S, Petrovic, J, Li, “A hybrid metaheuristic case-based reasoning system for nurse rostering”. Journal of Scheduling, vol 12(2), 2009, pp. 99-119, doi: 10.1007/s10951-008-0082-8.

[3] M. J. Bester, I. Nieuwoudt, \& J. H. Van Vuuren. (2007). Finding good nurse duty schedules: a case study. Journal of Scheduling, 10(6), 387-405.

[4] Burke, E. K., De Causmaecker, P., Vanden Berghe, G., \& Van Landeghem, H. (2004). The state of the art of nurse rostering. Journal of Scheduling, 7(6), 441-499.

[5] E. Corchado, D. MacDonald, and C. Fyfe, "Maximum and minimum likelihood hebbian learning for exploratory projection pursuit," Data Mining and Knowledge Discovery, vol. 8, May 2004, pp. 203-225,.

[6] E. Corchado and C. Fyfe, "Connectionist techniques for the identification and suppression of interfering underlying factors," International Journal of Pattern Recognition and Artificial Intelligence, vol. 17, Dec. 2003, pp. 1447-1466.

[7] A.T. Ernst, H. Jiang, M. Krishnamoorthy, B. Owens and D. Sier. An Annotated Bibliography of Personnel Scheduling and Rostering. Annals of Operations Research 127, 21-144, 2004. Kluwer Academic Publishers. Manufactured in The Netherlands.

[8] J. H. Friedman and J. W. Tukey, "A projection pursuit algorithm for exploratory data-analysis," IEEE Transactions on Computers, vol. 23, 1974, pp. 881-890.

[9] C. Fyfe, "A neural network for pca and beyond," Neural Processing Letters, vol. 6, 1997, pp. 33-41.

[10] C. Fyfe and E. Corchado, "Maximum likelihood hebbian rules," Proc. 10th European Symp. on Artificial Neural Networks (ESANN 2002), 2002, pp. 143-148. 
[11] Kempf, K., LePape, C., Smith, C., \& Fox, B. R. (1991). Issues in the design of AI-based schedulers: workshop report. Artificial Intelligence Magazine, 11, 37-46.

[12] A. Meisels, \& N. Lusternik. (1998). Experiments on networks of employee timetabling problems. In E. Burke \& M. Carter (Eds.), Lecture notes in computer science: Vol. 1408. Practice and theory of automated timetabling: selected papers from PATAT 1997 (pp. 130141). Berlin: Springer.

[13] Meyer auf'm H. Hofe, (2000). Solving rostering tasks as constraint optimization. In Lecture notes on computer science. Selected papers from the 3rd international conference on practice and theory of automated timetabling (PATAT) (pp. 280-297). Berlin: Springer.

[14] H. E. Miller, W. P. Pierskalla, \& G. J. Rath, (1976). Nurse scheduling using mathematical programming. Operations Research, 24(5), 857870.
[15] K., Miyashita \& K.Sycara, (1995). CABINS: A framework of knowledge acquisition and iterative revision for schedule improvement and reactive repair. Artificial Intelligence, 76, 377-426.

[16] E. Oja, "A simplified neuron model as a principal component analyzer," Journal of Mathematical Biology, vol. 15, 1982, pp. 267273,.

[17] D. Sanger, "Contribution analysis: a technique for assigning responsibilities to hidden units in connectionist networks," Connection Science, vol. 1, 1989, pp. 115-138.

[18] H. Hotelling, "Analysis of a complex of statistical variables into principal components,” Journal of Education Psychology, vol. 24, 1933, pp. 417-444.

[19] K. Pearson, "On lines and planes of closest fit to systems of points in space,” Philosophical Magazine, vol. 2, 1901, pp. 559-572.

[20] Seung, H. S., Socci, N. D., \& Lee, D. (1998). The Rectified Gaussian Distribution. Advances in Neural Information Processing Systems, 10, 350-356. 